\title{
STARLIKENESS FOR FUNCTIONS OF HYPERCOMPLEX VARIABLE
}

\author{
ANNA GORI AND FABIO VLACCI
}

\begin{abstract}
In this paper we introduce a new notion of starlikeness for a class of functions of hypercomplex variable and give several analytic equivalent conditions, which resemble the analogous ones in the holomorphic case. Furthermore we also give a characterization of starlikeness in terms of non-vanishing of a suitable analogue of Hadamard product which also generalizes the similar results in the complex analytic case.
\end{abstract}

Keywords: Regular functions of one quaternionic variable, starlikeness.

2010 Marhematics Subjecct Classification: 30G35, 30C45

\section{INTRODUCTION}

The notion of starlikeness has a central role in many different subjects of geometry and topology and is particularly important in geometric function theory. This very rich topic has been investigated in several papers (see [3] and the references therein) often following different approaches by many mathematicians, also in the hypercomplex setting (see [4],[5]). In the present paper we introduce a new definition of starlikeness for a class of functions of hypercomplex variable, which is inspired by a geometric point of view and which aims at providing tools for a generalization of the usual notions in the conformal and holomorphic setting.

Assume that $\mathbb{K}$ is an associative, unitary real algebra with division.

Definition 1.1. Assume $f$ is an injective slice-regular function in the unit ball $B(0,1)$ of $\mathbb{K}$ such that $f(0)=0$. Then we say that $f$ is starlike with respect to 0 if and only if, for any real $r$ such that $0 \leq r<1$, then $(1-t) f(B(0, r)) \subseteq f(B(0, r))$ for any real $t$ with $0 \leq t \leq 1$.

Since the class of functions we investigate can be regarded as (right) hypercomplex analytic functions, the characterization of the geometric aspects of starlikeness we introduce can be provided in terms of inequality conditions or using an approach involving the power series expansions.

In both cases, the results obtained generalize the corresponding ones in the complex holomorphic or analytic cases.

In particular, after applying the regular product $*$ as introduced in [8] for slice-regular functions, we are able to prove

\footnotetext{
Partially supported by Progetto MIUR di Rilevante Interesse Nazionale Proprietà geometriche delle
} varietà reali e complesse and by G.N.S.A.G.A (gruppo I.N.d.A.M). 
Proposition 1.2. Assume $f$ is a slice-regular starlike function in the unit ball $B(0,1)$ in $\mathbb{K}$, such that $f(0)=0$ then

$$
\Re e\left[\left(q f^{\prime}(q)\right) * f(q)^{-*}\right]>0
$$

Finally, with a suitable application of the Hadamard product $*_{H}$ in the hypercomplex case, if we denote by $\mathbb{S}_{\mathbb{K}}$ the sphere of imaginary units of $\mathbb{K}$, i.e. $\mathbb{S}_{\mathbb{K}}=\left\{q \in \mathbb{K}: q^{2}=-1\right\}$, we obtain this generalization of the result in [10]

Proposition 1.3. If a slice-regular function $f: B(0,1) \rightarrow \mathbb{H}$ such that $f^{\prime}(0)=1$ is starlike with respect to 0 then for any $s \in \mathbb{R}$ and for any $I \in \mathbb{S}_{\mathbb{K}}$ it turns out that

$$
q^{-1}\left[f(q) *_{H}\left[(1-q)^{-2}\left(q(1-s I)+q^{2} s I\right)\right] \neq 0\right.
$$

or equivalently, if $g$ is any primitive of $q^{-1} f(q)$,

$$
q^{-1}\left[g(q) *_{H}(1-q)^{-3}\left(q(1-s I)+q^{2}(1+s I)\right) \neq 0 .\right.
$$

Viceversa, if for a slice-regular function $f$ and any primitive $g$ of $q^{-1} f(q)$ conditions (1.2) or (1.3) hold, then $f$ is starlike with respect to 0.

\section{Background and Preliminary Results}

Let $\Omega$ be a topological vector space over $\mathbb{R}$.

Definition 2.1. A subset $E \subset \Omega$ is said to be starlike or star-shaped with respect to a point $w_{0} \in E$ if the linear segment joining $w_{0}$ to every other point $w \in E$ lies entirely in $E$. In formula, $E$ is starlike with respect to $w_{0} \in E$ if and only if, $\forall w \in E, \quad \forall t \in$ $\mathbb{R}, 0 \leq t \leq 1$, tw $+(1-t) w_{0}$ belongs to $E$.

Definition 2.2. A subset $E \subset \Omega$ is said to be convex if it is starlike with respect to each of its points; i.e. the linear segment joining any two points in E lies entirely in $E$.

In the present section we mainly deal with subsets of the topological vector spaces over $\mathbb{R}$ such as $\mathbb{R}^{N}$ or $\mathbb{C}^{N}$ for some $N \in \mathbb{N}$, which turn out to be also equipped with the standard Euclidean (Hermitian) product (respectively).

Definition 2.3. Let $f: X \rightarrow Y$ be a function and assume $Y$ is a topological vector space over $\mathbb{R}$. We say that $f$ is a

- starlike function if $f(X)$ is a starlike set in $Y$ (with respect to a point $f\left(x_{0}\right)$ );

- convex function if $f(X)$ is a convex set in $Y$.

Remark 2.4. The notion of starlikeness and convexity for sets or functions is invariant for rigid motions in a topological vector space.

Assume $X$ and $Y$ are open sets in $\mathbb{R}^{N}$ or $\mathbb{C}^{N}$ (for some $N \in \mathbb{N}$ ) such that $0 \in X$. We may always assume that $f(0)=0$, since otherwise one can consider $f_{1}=f-f(0)$. With this in mind, the condition of starlikeness for $f$ with respect to $0 \in f(X)$ can be also summarized in the following way 


$$
(1-t) f(X) \subseteq f(X) \quad \forall t \in[0,1]
$$

which allows us to use the following notation

$$
(1-t) f \prec f
$$

(commonly adopted for subordination relation of functions) and say that the function $h$, with $h(t, x)=(1-t) f(x)$, is subordinate to $f$.

If in addition we assume that $f: X \rightarrow Y$ is an injective starlike function (such that $f(0)=0)$ then the function

$$
\Phi_{t}(x):=f^{-1}((1-t) f(x))
$$

is well-defined; furthermore, if $f$ is a continuous function, so is $\Phi$. With $B(0, r)$ we indicate the open disc in $X$ centered at the origin of radius $r$, namely $B(0, r)=\{x \in$ $X:|x|<r\}$. We observe that $\Phi_{0}(x)=x$ for any $x \in X$ and if

$$
A_{r}:=f(X \cap B(0, r))
$$

for a positive $r$, then $(1-t) A_{r} \subseteq A_{r}$ and hence

$$
\Phi_{t}\left(A_{r}\right)=f^{-1}\left((1-t) A_{r}\right) \subseteq f^{-1}\left(A_{r}\right)=X \cap B(0, r) ;
$$

in other words, if $|x|=r_{1}<r$ and $x \in X$ then $\left|\Phi_{t}(x)\right| \leq r_{1}=|x|$. Furthermore, since for $t \neq 0,(1-t) A_{r} \subset \subset A_{r}$ it follows that $\left|\Phi_{t}(x)\right|=|x|$ if and only if $t=0$, which actually means $\Phi_{0}(x)=x$. Therefore we can summarize the previous considerations in the following

Lemma 2.5. If $h(t, x)=(1-t) f(x)$, is subordinate to $f$ and $f$ is injective in an open set of $\mathbb{R}^{N}$ or $\mathbb{C}^{N}$ (for some $N \in \mathbb{N}$ ) containing 0 and such that $f(0)=0$, then the function $\Phi_{t}(x):=f^{-1}((1-t) f(x))$ is well defined and it turns out that

$$
\left\|\Phi_{t}(x)\right\| \leq\|x\|
$$

where $\|\cdot\|$ is the (induced) Euclidean norm. Furthermore, equality in (2.4) holds if and only if $t=0$; in other words $\left\|\Phi_{t}(x)\right\|=\|x\|$ implies $\Phi_{t}(x)=x$ (which can actually occur if and only if $t=0$ ).

\section{The COMPleX HOlOMORPHiC CASE}

In this section we'll primarily consider the case of holomorphic starlike and convex functions in the complex plane. In particular, our attention will be focused on (normalized) holomorphic starlike and convex functions which are injective in $B(0,1)=\{z \in$ $\mathbb{C}:|z|<1\}$. The choice of this peculiar domain of definition is not restrictive, since, by the Riemann's Mapping Theorem, any simply-connected, open and connected set of $\mathbb{C}$ different from the entire complex plane $\mathbb{C}$ is biholomorphic to $B(0,1)$. 
We recall that in this setting an open and connected complex set is also called a domain of $\mathbb{C}$ and an injective holomorphic function is commonly said to be a univalent function or a schlicht function.

Finally, for our purposes, we can always assume that a univalent function $f: B(0,1) \rightarrow$ $\mathbb{C}$ is such that $f(0)=0$ and $f^{\prime}(0)=1$. Indeed, if $f$ is univalent (hence $f^{\prime}(0) \neq 0$ ) then so is

$$
z \mapsto \frac{f(z)-f(0)}{f^{\prime}(0)} .
$$

We then introduce the class of (normalized) functions

$\mathcal{S}:=\left\{f: B(0,1) \rightarrow \mathbb{C}, f\right.$ injective, holomorphic and such that $f(0)=0$ and $\left.f^{\prime}(0)=1\right\}$.

For univalent complex functions in $\mathcal{S}$, the notions of starlikeness and convexity with respect to 0 can be stated in a more analytic way, since one can apply the conformal properties of holomorphic functions to study the plane curves $t \mapsto \varrho e^{i t}$ and $t \mapsto f\left(\varrho e^{i t}\right)$ for suitable choices of radius $\varrho$.

We summarize the main results in the following (see e.g. [9])

Theorem 3.1. Given $f \in \mathcal{S}$, then

- $f$ is starlike with respect to 0 if and only if $\Re e\left\{\frac{z f^{\prime}(z)}{f(z)}\right\}>0$ for any $z \in B(0,1)$;

- $f$ is convex if and only if $\Re e\left\{1+\frac{z f^{\prime \prime}(z)}{f^{\prime}(z)}\right\}=\Re e\left\{\frac{\left(z f^{\prime}(z)\right)^{\prime}}{f^{\prime}(z)}\right\}>0$ for any $z \in B(0,1)$.

Additionally we might need the introduction of more refined definitions, namely for a real $\alpha$ with $0 \leq \alpha<1$

- $f$ is starlike with respect to 0 of order $\alpha$ if and only if $\Re e\left\{\frac{z f^{\prime}(z)}{f(z)}\right\}>\alpha>0$ for any $z \in B(0,1)$;

- $f$ is convex of order $\alpha$ if and only if $\Re e\left\{1+\frac{z f^{\prime \prime}(z)}{f^{\prime}(z)}\right\}=\Re e\left\{\frac{\left(z f^{\prime}(z)\right)^{\prime}}{f^{\prime}(z)}\right\}>\alpha>0$ for any $z \in B(0,1)$.

We observe that the condition of convexity for $f$ can be equivalently obtained from the conditon of starlikeness for the function $z \mapsto z f^{\prime}(z)$. We then introduce these classes of functions

$$
\begin{gathered}
\mathcal{S}^{*}:=\{f \in \mathcal{S}, \quad f \text { starlike with respect to } 0\} \quad \mathcal{C}:=\{f \in \mathcal{S}, \quad f \text { convex }\} \\
\mathcal{S}_{\alpha}^{*}:=\{f \in \mathcal{S}, \quad f \text { starlike with respect to } 0 \text { of order } \alpha\}
\end{gathered}
$$




$$
\mathcal{C}_{\alpha}:=\{f \in \mathcal{S}, \quad f \text { convex of order } \alpha\}
$$

Clearly

$$
\begin{gathered}
\mathcal{S}_{0}^{*}=\mathcal{S}^{*}, \quad \mathcal{S}_{\alpha}^{*} \subset \mathcal{S}^{*} \text { for positive } \alpha \\
\mathcal{C}_{0}=\mathcal{C}, \quad \mathcal{C}_{\alpha} \subset \mathcal{C} \text { for positive } \alpha
\end{gathered}
$$

and

$$
\mathcal{C} \subset \mathcal{S}^{*} \subset \mathcal{S}
$$

Furthermore we notice here that if $f \in \mathcal{S}^{*}$, then the function $\Phi$ as in (2.4) turns out to be holomorphic in $z$ and such that $\Phi_{t}(0)=0$ for any $t \in[0,1]$.

Since, traditionally, a holomorphic function with positive real part (or equivalently such that $\left.f(z) \in H_{+}:=\{z \in \mathbb{C}: \Re e(z) \geq 0\}\right)$ is known as a Carathéodory function, we introduce also the class of functions

$$
\mathcal{P}:=\left\{f: \mathbb{D} \rightarrow H_{+}, f \text { holomorphic and such that } f(0)=1\right\} .
$$

Therefore we can also say that a univalent function $f$ is such that

(1) $f \in \mathcal{S}^{*}$ if and only if $z \mapsto \frac{z f^{\prime}(z)}{f(z)}$ belongs to $\mathcal{P}$

(2) $f \in \mathcal{C}$ if and only if $z \mapsto 1+\frac{z f^{\prime \prime}(z)}{f^{\prime}(z)}=\frac{\left(z f^{\prime}(z)\right)^{\prime}}{f^{\prime}(z)}$ belongs to $\mathcal{P}$

(3) $f \in \mathcal{C}$ if and only if $z \mapsto 1+\frac{z f^{\prime \prime}(z)}{f^{\prime}(z)}-\alpha=\frac{\left(z f^{\prime}(z)\right)^{\prime}}{f^{\prime}(z)}-\alpha$ belongs to $\mathcal{P}$

(4) $f \in \mathcal{C}$ if and only if $z \mapsto z f^{\prime}(z)$ belongs to $\mathcal{S}^{*}$.

An interesting application of the classical Schwarz Lemma implies a geometric characterization for convex and starlike univalent functions, known as Theorem of Study (see [9])

Theorem 3.2. A univalent function $f: B(0,1) \rightarrow \mathbb{C}$ such that $f(0)=0$ is starlike with respect to 0 if and only if, for any real $r$ such that $0 \leq r \leq 1, f(B(0, r))$ is a starlike set with respect to 0 . A univalent function $f: B(0,1) \rightarrow \mathbb{C}$ such that $f(0)=0$ is convex if and only if, for any real $r$ such that $0 \leq r \leq 1, f(B(0, r))$ is a convex set.

Similarly, it follows directly from Schwarz Lemma that, whenever $f$ is a univalent function from the unit disc $B(0,1)$ to $\mathbb{C}$ and starlike with respect to 0 , then $\Phi(t, z)=$ $f^{-1}((1-t) f(z))$ is a Schwarz function for any $t$, with $0 \leq t \leq 1$, namely for any given $t$, with $0 \leq t \leq 1$ the function $\Phi(t, z)$ is holomorphic in $B(0,1)$ and

$$
\Phi(t, 0)=0 \quad \text { and } \quad|\Phi(t, z)| \leq|z| \quad \forall z \in B(0,1)
$$




\section{The AnAlytic CASE}

Given two analytic functions $f, g$ whose power series are

$$
f(w)=\sum_{n} w^{n} a_{n}, \quad g(w)=\sum_{n} w^{n} b_{n}
$$

where $a_{n}$ and $b_{n}$ belong to a ring $(\mathcal{R},+, \cdot)$, we say that $f=g$ if and only if $a_{n}=b_{n}$ for any $n$. Furthermore, we can define the Hadamard product of the analytic functions $f$ and $g$ in the following way

$$
f *_{H} g(w):=\sum_{n} w^{n} a_{n} \cdot b_{n}
$$

It turns out that the Hadamard product of analytic functions is an analytic function. In general the Hadamard product is not commutative, however if the ring $\mathcal{R}$ is a unitary ring of unit $1_{\mathcal{R}}$ for $\cdot$, the function $w \mapsto \sum_{n} w^{n} \cdot 1_{\mathcal{R}}$ is the neutral element for the Hadamard product. It will be also denoted by $(1-w)^{-1}$. Furthermore if the ring is a field, then any function $f(w)=\sum_{n} w^{n} a_{n}$ with $a_{n}$ different from the neutral element $0_{\mathcal{R}}$ for + in $\mathcal{R}$, has an inverse for the Hadamard product given by

$$
w \mapsto \sum_{n} w^{n}\left(a_{n}\right)^{-1}
$$

In addition to that, if $f(w)=\sum_{n} w^{n} a_{n}$ we consider the following operations for analytic functions:

- $w^{p} f(w)=\sum_{n} w^{(n+p)} a_{n}$ with $p \in \mathbb{Z}$

- $f^{\prime}(w)=\sum_{n} w^{n-1} n a_{n}$ where $n a_{n}=\underbrace{a_{n}+a_{n}+\ldots+a_{n}}_{n \text {-times }}$

- $a f(w)=\sum_{n} w^{n}\left(a \cdot a_{n}\right)$ with $a \in \mathcal{R}$.

Remark 4.1. The function $f^{\prime}$ is said to be derived from the function $f$ or to be the derivative of $f$

It follows that

$$
\begin{gathered}
\left(a++_{\mathcal{R}} b\right) f(w)=a f(w)+b f(w), \text { with } a, b \in \mathcal{R} . \\
w f^{\prime}(w) *_{H} g(w)=f(w) *_{H} w g^{\prime}(w)
\end{gathered}
$$

and

$$
\left(w f^{\prime}(w)\right)^{\prime}=f^{\prime}(w)+w\left(f^{\prime}\right)^{\prime}(w) .
$$

Therefore if $a_{0}=0_{\mathcal{R}}$ and $a_{1}=1_{\mathcal{R}}$ then

$$
\left(w f^{\prime}(w)\right)^{\prime}=f^{\prime}(w)+w\left(f^{\prime}\right)^{\prime}(w)=f^{\prime}(w) *_{H}\left(\sum_{n} w^{n-1} n 1_{\mathcal{R}}\right)
$$

and hence there is no $k \in \mathcal{R}$ such that 


$$
\left(w f^{\prime}(w)\right)^{\prime}=k f^{\prime}(w)
$$

Furthermore, given $f(w)=\sum_{n} w^{n} a_{n}$, if, for any $n$ there exists $c_{n}$ such that

$$
\underbrace{c_{n}+c_{n}+\ldots+c_{n}}_{(n+1)-\text { times }}=a_{n}
$$

then $F(w)=\sum_{n} w^{n+1} c_{n}$ has the property

$$
F^{\prime}(w)=f(w)
$$

and the function $F$ is called a primitive of $f$.

\section{THE REgular CASE}

Assume that $\mathbb{K}$ is an associative, unitary real algebra with division. As for the case $\mathbb{K}=\mathbb{C}$, if $q \in \mathbb{K}$ we denote by $\Re e q$ the real part of $q$; in the same way, the conjugate of $q$ will be denoted by $\bar{q}:=2 \Re e q-q$.

Each algebra $\mathbb{K}$ can be regarded as a normed topological vector space, after introducing $|q|^{2}=q \bar{q} \in \mathbb{R}$ as the norm of an element $q$ of the algebra. Therefore if $q \neq 0$, then $q^{-1}=|q|^{-2} \bar{q}$; since the center of $\mathbb{K}$ coincides with $\mathbb{R}$ there is no ambiguity in writing $q^{-1}$ as $|q|^{-2} \bar{q}$ or as $\bar{q}|q|^{-2}$ so we often adopt the notation $\bar{q} /|q|^{2}$ which will be applied in general for a fraction when the divisor is a real number (different from zero).

In this setting it is possible (see [8])) to introduce a notion of regularity for functions defined in any open ball $B(0, r)=\{q \in \mathbb{K}:|q|<r\}$ (and, more in general, in some axially symmetric domain) of $\mathbb{K}$ which corresponds to the one of holomorphicity in the complex case. For this class of regular functions, called slice-regular functions, most of the results valid for holomorphic functions are extended, but many other new phenomena may occur (the interested reader could refer to [8]). In particular sliceregular functions are characterized to be analytic functions with coefficients on (say) the right; namely $f$ is slice-regular in $B(0, r) \subset \mathbb{K}$ if and only if there exists a converging power series $\sum_{n} q^{n} a_{n}$ with $a_{n} \in \mathbb{K}$ for any $n \in \mathbb{N}$ such that

$$
f(q)=\sum_{n} q^{n} a_{n}
$$

The notions of derivative (and primitive) functions can be naturally introduced for slice-regular functions, therefore, this class of functions perfectly fits for our purpouses.

For the sequel it will be important the introduction of a "regularized" multiplication between converging regular power series, i.e. representing a slice-regular function.

Definition 5.1. Let $f(q)=\sum_{n=0}^{+\infty} q^{n} a_{n}$ and $g(q)=\sum_{n=0}^{+\infty} q^{n} b_{n}$ be given power series with coefficients in $\mathbb{K}$ whose radii of convergence are greater than $R$. We define the regular product of $f$ and $g$ as the series $f * g(q)=\sum_{n=0}^{+\infty} q^{n} c_{n}$, where $c_{n}=\sum_{k=0}^{n} a_{k} b_{n-k}$ for all $n$. 
It is not difficult to see that $f * g$ is a slice-regular function defined in the open ball $B(0, R)$. Furthermore, the regular product is extended for slice-regular functions defined on a general axially symmetric domain ${ }^{1} \Omega$ in the following way

$$
f * g(q)=\left\{\begin{array}{cl}
0 & \text { if } f(q)=0 \\
f(q) g\left(f(q)^{-1} q f(q)\right) & \text { otherwise. }
\end{array}\right.
$$

Remark 5.3. Note that the regular product of two power series is completely different from the Hadamard product.

Definition 5.4. For a regular function $f: B(0, R) \rightarrow \mathbb{K}$ having power series expansion $\sum_{n=0}^{+\infty} q^{n} a_{n}$ we define the regular conjugate $f^{c}$ and the symmetrization or symmetrized $f^{s}$ of $f$ as

$$
\begin{gathered}
f^{c}(q)=\sum_{n=0}^{+\infty} q^{n} \bar{a}_{n} \\
f^{s}(q)=f * f^{c}(q)=f^{c} * f(q)=\sum_{n=0}^{+\infty} q^{n} r_{n}
\end{gathered}
$$

with $r_{n}=\sum_{k=0}^{n} a_{k} \bar{a}_{n-k} \in \mathbb{R}$.

Definition 5.5. Let $f: B(0, R) \rightarrow \mathbb{K}$ be a slice-regular function and suppose $f \not \equiv 0$. Then the regular reciprocal of $f$ is the (slice-regular) function $f^{-*}=\frac{1}{f^{s}(q)} f^{c}$ defined on $B \backslash Z\left(f^{s}\right)$, where $Z\left(f^{s}\right)$ is the zero set of the symmetrized function $f^{s}$.

Regular reciprocals are well-defined slice-regular functions whose power series expansions are converging in their domains of definition.

Recently, some attempts to generalize the notion of convexity and starlikeness from the holomorphic case to the class of quaternionic valued functions have appeared (see $[4],[5],[6])$. In this paper we follow a different approach and give the following

Definition 5.6. Assume $f$ is an injective slice-regular function in the unit ball of $\mathbb{K}$ such that $f(0)=0$. Then we say that $f$ is starlike with respect to 0 if and only if, for any real $r$ such that $0 \leq r<1$, then $(1-t) f(B(0, r)) \subseteq f(B(0, r))$ for any real $t$ with $0 \leq t \leq 1$.

\footnotetext{
${ }^{1}$ If we denote by $\mathbb{S}_{\mathbb{K}}$ the sphere of imaginary units of $\mathbb{K}$, i.e. $\mathbb{S}_{\mathbb{K}}=\left\{q \in \mathbb{K}: q^{2}=-1\right\}$, then every non real element $q$ can be written in a unique way as $q=x+y I_{q}$, with $I_{q} \in \mathbb{S}_{\mathbb{K}}$ and $x, y \in \mathbb{R}, y>0$. We will refer to $x=\Re e(q)$ as the real part of $q$ and $y=\operatorname{Im}(q)$ as the imaginary part of $q$.

Definition 5.2. Let $\Omega \subseteq \mathbb{K}$ be a domain in $\mathbb{K}$; we say that $\Omega$ is an axially symmetric domain if, for all $x+I y \in \Omega$, the whole sphere $x+\mathbb{S}_{\mathbb{K}} y$ is contained in $\Omega$.
} 
In analogy with the complex holomorphic case we introduce the following classes of slice-regular functions

$\mathcal{S}_{\mathbb{K}}:=\left\{f: B(0,1) \rightarrow \mathbb{K}, f\right.$ injective, slice - regular and such that $f(0)=0$ and $\left.f^{\prime}(0)=1\right\}$.

$$
\begin{gathered}
\mathcal{S}_{\mathbb{K}}^{*}:=\{f: B(0,1) \rightarrow \mathbb{K}, \text { slice }- \text { regular starlike with respect to } 0\} \\
\mathcal{P}_{\mathbb{K}}:=\{f: B(0,1) \rightarrow \mathbb{K}, f \text { slice }- \text { regular such that } \Re e f>0 ; f(0)=1\} .
\end{gathered}
$$

5.1. Inequality conditions for starlikeness. Despite the similarity with the complex holomorphic case, the assumption of starlikness allows us only to define the function $\Phi(t, q)=f^{-1}((1-t) f(q))$ for any real $t$ with $0 \leq t \leq 1$ when $q \in \mathbb{K}$, but in general one cannot prove any regularity (beside continuity) for $\Phi(t, q)$ in $q$ (for a given $t$, with $0 \leq t \leq 1$ ) even though $f$ is assumed to be a slice-regular function. However, also in the complex holomorphic case, one has to ask for conditions on regularity with respect to $t$; so in this setting we need to prove a lemma which generalizes the one in [11] for holomorphic functions.

Lemma 5.7. With the above given notations (and assumptions) for $f \in \mathcal{S}_{\mathbb{K}}^{*}$ and $\Phi$, assume there exists a positive @ such that the limit

$$
\lim _{t \rightarrow 0^{+}} \frac{\Phi(0, q)-\Phi(t, q)}{t^{\varrho}}
$$

exists; call it $\omega_{\varrho}(q)$. Then, for $q \neq 0$,

$$
\Re e\left(q^{-1} \omega_{\varrho}(q)\right) \geq 0 .
$$

Proof. Define, for $q \neq 0$,

$$
\Psi(t, q):=2 q(q+\Phi(t, q))^{-1}(q-\Phi(t, q)) ;
$$

it follows from Lemma 2.5 that the function $\Psi$ is well-defined.

Furthermore, $q^{-1} \Psi(t, q)=2(q+\Phi(t, q))^{-1}(q-\Phi(t, q))$ and hence

$$
\begin{gathered}
\Re e\left(q^{-1} \Psi(t, q)\right)=2 \Re e\left((q+\Phi(t, q))^{-1}(q-\Phi(t, q))=\right. \\
=2 \Re e\left(\left(q\left(1+q^{-1} \Phi(t, q)\right)^{-1}\left(q\left(1-q^{-1} \Phi(t, q)\right)\right)=\right.\right. \\
=2 \Re e\left(\left(1+q^{-1} \Phi(t, q)\right)^{-1}\left(1-q^{-1} \Phi(t, q)\right)\right) . \\
9
\end{gathered}
$$


Put $\left.\alpha:=q^{-1} \Phi(t, q)\right)$; since

$$
\begin{gathered}
\Re e\left(q^{-1} \Psi(t, q)\right)=2 \Re e\left((1+\alpha)^{-1}(1-\alpha)\right)= \\
=\frac{(1+\bar{\alpha})(1-\alpha)+(1+\alpha)(1-\bar{\alpha})}{|1+\alpha|^{2}}=2 \frac{1-|\alpha|^{2}}{|1+\alpha|^{2}}
\end{gathered}
$$

from (2.4) we conclude that

$$
\Re e\left(q^{-1} \Psi(t, q)\right) \geq 0
$$

Finally we have

$$
\begin{gathered}
\lim _{t \rightarrow 0^{+}} \frac{q^{-1} \Psi(t, q)}{t^{\varrho}}=\lim _{t \rightarrow 0^{+}} \frac{q^{-1} 2 q(q+\Phi(t, q))^{-1}(q-\Phi(t, q))}{t^{\varrho}}= \\
=\lim _{t \rightarrow 0^{+}}\left[2(q+\Phi(t, q))^{-1} \frac{(q-\Phi(t, q))}{t^{\varrho}}\right] ;
\end{gathered}
$$

we recall that $\Phi(0, q)=q$ for any $q$, therefore, using the assumption on the existence of $\lim _{t \rightarrow 0^{+}} \frac{(q-\Phi(t, q))}{t^{\varrho}}=\omega_{\varrho}(q)$ and (5.3) we obtain that

$$
\Re e\left(q^{-1} \omega_{\varrho}(q)\right) \geq 0 .
$$

Let $H(t, q):=(1-t) f(q)$ then

$$
H(t, q)-H(0, q)=-t f(q) .
$$

Therefore, $H(t, q)-H(0, q)$ is a slice-regular function for any $t$; moreover there exists the limit

$$
\lim _{t \rightarrow 0^{+}} \frac{H(t, q)-H(0, q)}{t}=-f(q) .
$$

Now $f(\Phi(t, q))=H(t, q)$ and $f(\Phi(t, q))-f(q)=H(t, q)-H(0, q)$. On the other hand as in $[2]$

with

$$
f(\Phi(t, q))-f(q)=(\Phi(t, q)-q) * R_{\Phi(t, q)}^{f}(q)
$$

$$
\lim _{t \rightarrow 0^{+}} R_{\Phi(t, q)}^{f}(q)=f^{\prime}(q)
$$

So

$$
\lim _{t \rightarrow 0^{+}} \frac{f(q)-f(\Phi(t, q)))}{t}=f(q)=\lim _{t \rightarrow 0^{+}} \frac{q-\Phi(t, q)}{t} * R_{\Phi(t, q)}^{f}(q)=\omega_{1}(q) * f^{\prime}(q)
$$

where, from Lemma 5.7, $\Re e q^{-1} \omega_{1}(q) \geq 0$. With this in mind we conclude that

$$
\begin{aligned}
0 & \leq \Re e\left[q^{-1} f(q) * f^{\prime}(q)^{-*}\right]=\Re e\left[f(q) * q^{-1} * f^{\prime}(q)^{-*}\right]= \\
& =\Re e\left[f(q) *\left(f^{\prime}(q) * q\right)^{-*}\right]=\Re e\left[f(q) *\left(q f^{\prime}(q)\right)^{-*}\right]
\end{aligned}
$$


Note that $f^{\prime}(q) \neq 0$ due to injectivity of $f$. Moreover observe that the real part of a slice-regular function satisfies the maximum/minimum principle (see [8] p.108) hence the previous inequality is strict.

We can moreover assert that the function $\left(q f^{\prime}(q)\right) * f(q)^{-*} \in \mathcal{P}_{\mathbb{K}}$ i.e.

$$
\Re e\left[\left(q f^{\prime}(q)\right) * f(q)^{-*}\right]>0
$$

which is in complete analogy with the complex holomorphic case. This follows from

Lemma 5.8. Let $\Omega$ be an axially symmetric domain and let $f: \Omega \rightarrow \mathbb{K}$ be a slice-regular function. If $\Re$ ef $>0$ in $\Omega$ then $\Re e\left(f^{-*}\right)$ in $\Omega^{\prime}:=\Omega \backslash Z\left(f^{s}\right)$.

Proof. The function $f^{-*}$ is well defined on $\Omega^{\prime}$, where the following formula holds

$$
f^{-*}(q)=f(T(q))^{-1}
$$

with $T(q):=f^{c}(q)^{-1} q f^{c}(q)$ which sends $\Omega^{\prime}$ to itself. Now recalling that $q^{-1}=|q|^{-2} \bar{q}$ we obtain

$$
\Re e\left(f^{-*}(q)\right)=\Re e\left(f(T(q))^{-1}\right)=|f(T(q))|^{-2} \Re e(f(T(q)))>0 .
$$

We have therefore proved

Proposition 5.9. Assume $f$ is in $\mathcal{S}_{\mathbb{K}}^{*}$ then

$$
q^{-1} f(q) * f^{\prime}(q)^{-*} \in \mathcal{P}_{\mathbb{K}} .
$$

or analogously

$$
q f^{\prime}(q) * f(q)^{-*} \in \mathcal{P}_{\mathbb{K}}
$$

We can also prove the converse of the previous proposition, namely

Proposition 5.10. Assume that $f$ is in $\mathcal{S}_{\mathbb{K}}$ and is such that $q^{-1} f(q) * f^{\prime}(q)^{-*} \in \mathcal{P}_{\mathbb{K}}$. Then $f$ belongs to $\mathcal{S}_{\mathbb{K}}^{*}$.

Proof. Take $q \in B(0, r)$ and consider $(1-t) f(q)$. For $|t|<\varepsilon$, with $\varepsilon$ sufficiently small, there exists $v(t, q) \in B(0, r)$ such that $f(v(t, q))=(1-t) f(q)$ since $f$ is a local diffeomorphism. Observe that $v(0, q)=q$ from the univalence of $f$. Now, as proved in [2], we have

$$
f(v(t, q))-f(v(0, q))=-t f(q)=(v(t, q)-q) * R_{v(t, q)}^{f}(q)
$$

thus

$$
v(t, q)-q=-t f(q) * R_{v(t, q)}^{f}(q)^{-*}
$$

and

Therefore

$$
\left.\frac{\partial v}{\partial t}(t, q)\right|_{t=0}=-f(q) * f^{\prime}(q)^{-*}
$$

$$
v(t, q)=v(0, q)-\underset{11}{f}(q) * f^{\prime}(q)^{-*} t+g(t)
$$


where $\lim _{t \rightarrow 0^{+}} \frac{g(t)}{t}=0$.

Using the assumption on the positivity of the real part of $q^{-1} f(q) * f^{\prime}(q)^{-*}$ we get that $|v(t, q)|$ is decreasing in $t$ in an open neighborhood of 0 . In fact

$$
\begin{gathered}
\lim _{t \rightarrow 0^{+}} \frac{|v(t, q)|^{2}-|v(0, q)|^{2}}{t}= \\
=\lim _{t \rightarrow 0} \frac{\left.\left(\overline{v(0, q)}-\overline{f(q) * f^{\prime}(q)^{-*} t}-\overline{g(t)}\right)\left(v(0, q)-f(q) * f^{\prime}(q)^{-*} t+g(t)\right)\right)-|q|^{2}}{t}= \\
=-2 \Re e \bar{q} f(q) * f^{\prime}(q)^{-*}=-2|q|^{2} \Re e q^{-1} f(q) * f^{\prime}(q)^{-*} \leq 0 .
\end{gathered}
$$

Hence each point of the ray $(1-t) f(q)$ for $0<t \leq 1$ is the image of a point $v(t, q) \in$ $B(0, r)$ for each $q$ such that $|q| \leq r \leq 1$. We conclude that, for any $r<1, f(B(0, r))$ is starlike with respect to 0 , so $f$ is starlike.

5.2. Hadamard conditions for starlikeness. Assume $f$ is in $\mathcal{S}_{\mathbb{K}}^{*}$ and $f^{\prime}(0)=1$. Under these hypotheses, the (slice-regular) function $q^{-1} f(q)$ is well defined, since if $f(q)=\sum_{n \geq 1} q^{n} a_{n}$ then

$$
q^{-1} f(q)=\sum_{n \geq 1} q^{n-1} a_{n}
$$

Let $g$ be a primitive of $q^{-1} f(q)$; in other words, if $g(q)=\sum_{n \geq 1} q^{n} b_{n}$ then $g^{\prime}(q)=q^{-1} f(q)$. This condition implies that $n b_{n}=a_{n}$ for any $n \geq 0$. In particular, since $a_{0}=0$ and $a_{1}=1$ then $b_{0}=0$ and $b_{1}=1$.

Definition 5.11. We say that a slice-regular function $g$ in the unit ball, such that $g(0)=0$ and $g^{\prime}(0)=1$ is convex iff

$$
\Re e\left(\left(q g^{\prime}\right)^{\prime}(q) * g^{\prime}(q)^{-*}\right)>0 .
$$

We have the following results which generalizes the one in $[10]$

Lemma 5.12. If a function $f$ is in $\mathcal{S}_{\mathbb{K}}^{*}$ and $f^{\prime}(0)=1$ then any given primitive $g$ of $q^{-1} f(q)$ is convex.

Proof. Since

$$
\begin{gathered}
\Re e\left(\left(q g^{\prime}(q)\right)^{\prime} * g^{\prime}(q)^{-*}\right)=\Re e\left(1+q g^{\prime \prime}(q) * g^{\prime}(q)^{-*}\right)= \\
=\Re e\left(1+\left(q f^{\prime}(q)-f(q)\right) * f(q)^{-*}\right)=\Re e\left(q f^{\prime}(q) * f(q)^{-*}\right)>0
\end{gathered}
$$

we conclude that $g$ is convex.

Proposition 5.13. If a function $f$ is in $\mathcal{S}_{\mathbb{K}}^{*}$ and $f^{\prime}(0)=1$ then for any $s \in \mathbb{R}$ and for any $I \in \mathbb{S}_{\mathbb{K}}$ it turns out that

$$
q^{-1}\left[f(q) *_{H}\left[(1-q)^{-2}\left(q(1-s I)+q^{2} s I\right)\right] \neq 0\right.
$$


or equivalently, if $g$ is any primitive of $q^{-1} f(q)$,

$$
q^{-1}\left[g(q) *_{H}(1-q)^{-3}\left(q(1-s I)+q^{2}(1+s I)\right) \neq 0 .\right.
$$

Viceversa, if for a slice-regular function $f$ and any primitive $g$ of $q^{-1} f(q)$ conditions (5.6) or (5.7) hold, then $f \in \mathcal{S}_{\mathbb{K}}^{*}$ and $g$ is convex.

Proof. Note that

$$
\begin{aligned}
& q^{-1}\left[g(q) *_{H}(1-q)^{-3}\left(q(1-s I)+q^{2}(1+s I)\right)=\right. \\
= & q^{-1}\left[g(q) *_{H} q(1-q)^{-3}((1-s I)+q(1+s I))=\right. \\
= & q^{-1}\left[g(q) *_{H} q(1-q)^{-3}(2 q(1+q) s I+(1-q)(1-s I+2 q s I))=\right. \\
= & q^{-1}\left[g ( q ) * _ { H } q ( 1 - q ) ^ { - 3 } \left(2 q(1+q) s I+q^{-1}\left[g(q) *_{H} q(1-q)^{-2}(1-s I+2 q s I)\right)=\right.\right. \\
= & q^{-1}\left[g(q) *_{H} q\left[(1-q)^{-2}\left(q(1-s I)+q^{2} s I\right)\right]^{\prime}\right]
\end{aligned}
$$

Using equation (4.2) it then follows that

$$
\begin{aligned}
& q^{-1}\left[g(q) *_{H} q\left[(1-q)^{-2}\left(q(1-s I)+q^{2} s I\right)\right]^{\prime}\right]= \\
= & q^{-1}\left[q g^{\prime}(q) *_{H}\left[(1-q)^{-2}\left(q(1-s I)+q^{2} s I\right)\right]=\right. \\
= & q^{-1}\left[f(q) *_{H}\left[(1-q)^{-2}\left(q(1-s I)+q^{2} s I\right)\right],\right.
\end{aligned}
$$

so we conclude that condition (5.6) is equivalent to condition (5.7).

Now from the starlikeness of $f$ it turns out that $g$ is convex, then

$$
\Re e\left[\left(q g^{\prime}\right)^{\prime}(q) *\left(g^{\prime}(q)\right)^{-*}\right]>0 ;
$$

from normality conditions on $f$ it also follows that

$$
\left.\left(q g^{\prime}\right)^{\prime}(q) *\left(g^{\prime}(q)\right)^{-*}\right|_{q=0}=1
$$

and from

$$
g(q)=q+\sum_{n \geq 2} q^{n} b_{n}
$$

we have

$$
\begin{gathered}
\left(q g^{\prime}\right)^{\prime}(q)=1+\sum_{n \geq 2} n^{2} q^{n-1} b_{n}= \\
=g^{\prime}(q) *_{H} \sum_{n \geq 1} n q^{n-1}=g^{\prime}(q) *_{H}(1-q)^{-2} .
\end{gathered}
$$

Hence, 


$$
\Re e\left[\left(q g^{\prime}\right)^{\prime}(q) *\left(g^{\prime}(q)\right)^{-*}\right]>0
$$

is equivalent to

$$
\left(q g^{\prime}\right)^{\prime}(q) *\left(g^{\prime}(q)\right)^{-*} \neq s I
$$

for any $s \in \mathbb{R}$ and for any $I \in \mathbb{S}_{\mathbb{K}}$ or to

$$
\left(q g^{\prime}\right)^{\prime}(q)+g^{\prime}(q) s I \neq 0
$$

which can be also written as

$$
g^{\prime}(q) *_{H}\left((1-q)^{-2}+(1-q)^{-1} s I\right) \neq 0
$$

thanks to associativity property of the Hadamard product and the notation adopted for the neutral element of Hadamard product. Finally, from

$$
g^{\prime}(q) *_{H}\left((1-q)^{-2}+(1-q)^{-1} s I\right)=g^{\prime}(q) *_{H}\left((1-q)^{-2}\left(q(1-s I)-q^{2} s I\right)\right.
$$

we conclude our proof.

The authors are also looking for possible new characterizations of other classes of functions of hypercomplex variable and for their applications to the proof of other statements. In this sense, a version of the solution of the Bieberbach conjecture for starlike functions of hypercomplex variable will be given in a forthcoming paper.

\section{REFERENCES}

[1] R.W. Barnard, A Generalization of Study's Theorem on Convex Maps, Proc. Amer. Math. Soc., 72 (1), 127-134, (1978).

[2] C. Bisi, C. Stoppato, The Schwarz-Pick lemma for slice regular functions, Indiana Univ. Math. J., 61, 297-317 (2012).

[3] P. Duren, Univalent functions, 259. Springer-Verlag, New York, 1983.

[4] S. GAL, Elements of Geometric Theory of functions of quaternionic variable, Adv. Appl. Clifford Algebras, 10, 91-106 (2000).

[5] S. GAL, Starlike, convex and alpha-convex functions of hyperbolic complex and of dual complex variable, Studia Univ. Babel-Bolyai Math., 46, 23-40 (2001).

[6] S. Gal, J. O. Gonzàlez-Cervantes, I. Sabadini, On Some Geometric Properties of Slice Regular Functions of Quaternion Variable, arXiv:1410.2623

[7] G. Gentili, C. Stoppato, D. Struppa, Regular functions of a quaternionic variable, Springer Monographs in Mathematics, Springer, Heidelberg, 2013.

[8] G. Gentili, D. Struppa, A new theory of regular functions of a quaternionic variable, Adv. Math., 216, 279-301 (2007).

[9] R. Remmert, Theory of complex functions, Graduate Texts in Mathematics, 122, Springer-Verlag, New York, 1991.

[10] H. Silverman, E.M. Silvia, D. Telage, Convolution Conditions for Convexity, Starlikeness and Spiral-Likeness, Math. Z., 162, 125-130, (1978).

[11] T. J. Suffridge, The principle of subordination applied to functions of several variables, Pacific J. Math., 33, 241-248 (1970). 
Dipartimento di Matematica - Università di Milano, Via Saldini 50, 20133 Milano, ITALY

E-mail address: anna.gori@unimi.it

Dipartimento di Matematica "U. Dini" - Università di Firenze, Viale Morgagni 67/A, 50134 FiRENZE, ITALY

E-mail address: vlacci@math.unifi.it 